$\mathbb{T}$ periodica polytechnica

\author{
Transportation Engineering \\ $38 / 1(2010) 53 \sqrt{60}$ \\ doi: 10.3311/pp.tr.2010-1.09 \\ web: http://www.pp.bme.hu/tr \\ (c) Periodica Polytechnica 2010
}

RESEARCH ARTICLE

\section{Part failure diagnosis for internal combustion engine using noise and vibration analysis}

\author{
Pál Bánlaki / Zoltán Magosi
}

Received 2009-10-01

\begin{abstract}
Former practice proves that the vibration and noise caused by rotating or alternately moving parts together with the physical and chemical processes inside an internal combustion engine contain a great amount of information about what happens inside the machine. The art of extracting useful information from time based vibro-acoustic signals is especially difficult in the case of an operating internal combustion engine because there are many vibration and noise sources and the rotation speed is not constant. The other problem is that the rotational speed is not constant in the case of an internal combustion engine during a single working cycle and in the course of continuous operation. we developed a method to follow the fine details of angular speed fluctuations e. g. because of the activity of single piston. Our purpose was to develop a flexible and improvable measurement system which is usable both for experiments and final tests of several engines. Using the measuring set-up we could exploit the order analysis function together with the analysis of results in the frequency domain.
\end{abstract}

\section{Keywords}

noise and vibration diagnostics - order analysis - internal combustion engine

\section{Acknowledgement}

Authors are grateful for the support of GMPTH Szentgotthárd and express their thanks personally to our helpful colleges: Pál Henter, Szilveszter Kulcsár, Csaba Pál, and József Mesics.

Also, many thanks for the the staff of NI Hungary, especially the kind support from Attila Péter, Tamás Sárosi and Márton Litkei.

\section{Pál Bánlaki}

Department of Vehicle Manufacturing and Repairing, BME, Bertalan L. u. 2., H-1111 Budapest, Hungary

\section{Introduction}

Our former experiments and observations confirm that vibration and noise analysis is an effective method to qualify internal combustion engines as one of the measurement processes during the so called cold test. In the course of the cold test an electric motor drives the engine. The noise and vibration data collection can be executed at constant rotation speeds. As a consequence the classic FFT method is usable. The analysis of the spectral image of the engine behavior gives us the possibility to screen the problematic or the doubtful products. Currently the method is highly reliable and effective, and allows assessment of assembly faults, damaged parts and other abnormalities. Several windowing methods render possible the automatic computer based data evaluation and decision making, too. To demonstrate the method and form an initial idea, the figures below (Figs. 1. 2) show the frequency domain spectrum diagrams of a perfect engine and a faulty engine [1].

In many cases even the faulty parts or fault location is determinable based on a formerly collected and assembled fault knowledge base [9]. E.g. the automatic, computer based screening procedure is capable and used in practice to catch deformed plastic covers of incorrect shape, which cause a soft characteristic noise when get in touch eventually with the moving belt underneath.

Naturally, there is a big demand to use noise and vibration diagnosis for analysis of internal combustion engines operating under normal working conditions with fuel. However, if the rotational speed is not constant, the traditional method using data collection on time base (by fixed sample intervals), namely Fourier transformation to switch over to frequency domain followed by information evaluation is not usable. Because of the continuous variation of the base frequency, obtainment of usable results for the frequency domain image is not possible as the so called leakage effect makes the result almost meaningless.

A relative new measurement technique called order analysis is the secret of sorting out all the many signal components that a machine with variations in the rotation speed can generate. 


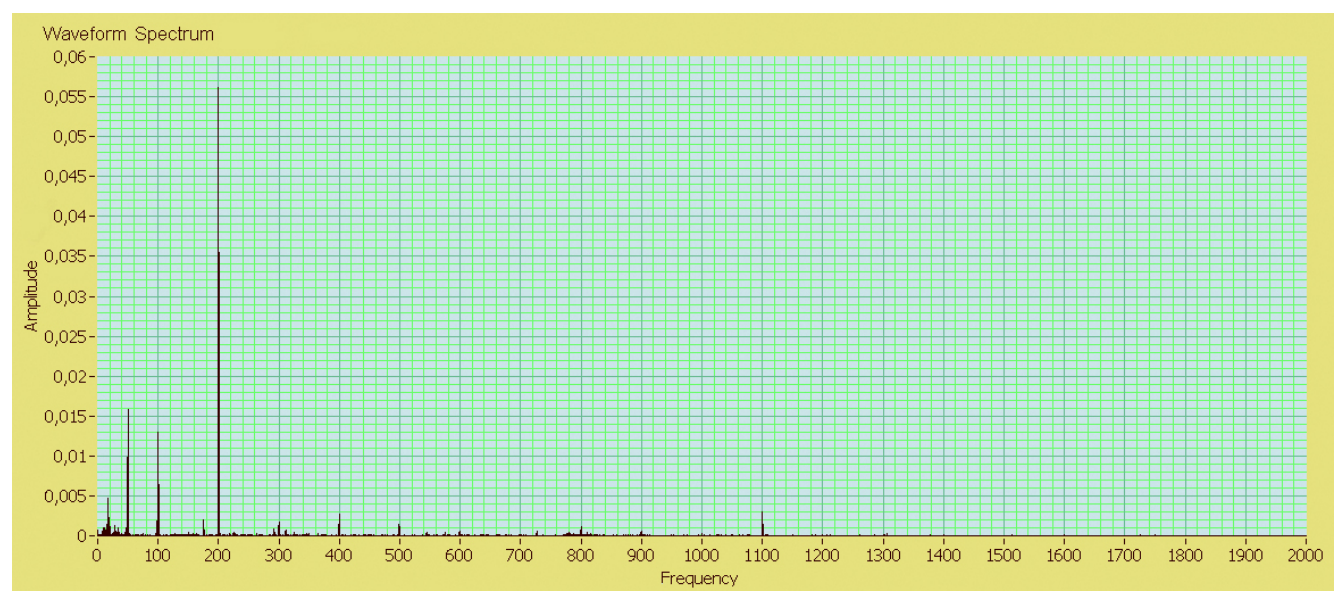

Fig. 1. Noise spectrum of a perfect engine

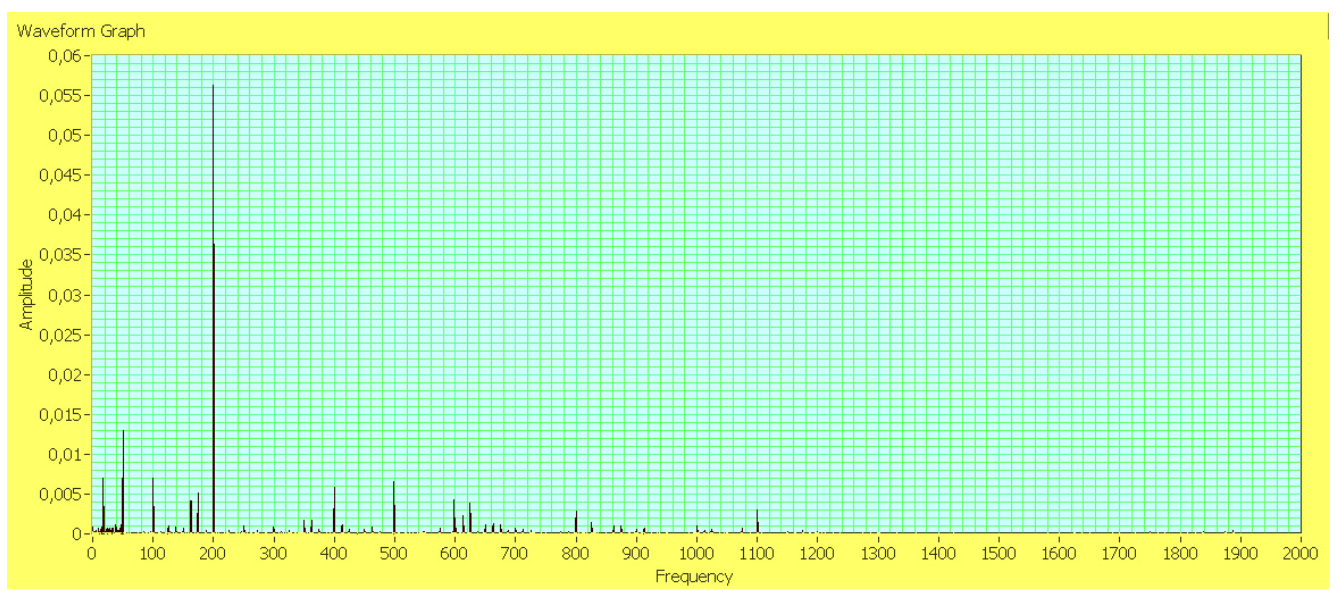

Fig. 2. Noise spectrum of a faulty engine.

\section{Order analysis principles and the measuring set-up}

The traditional FFT process transforms time domain data to frequency domain, creating a spectrum. Signals that are periodic (repetitive) in the time domain appear as some peaks in the frequency domain. If the angular or rotation speed is not constant, the signals are not really periodic and there will be not well observable peaks in the frequency domain. The new great idea is that instead of collecting data by given time intervals in time domain one must collect by given angular intervals in revolution domain [2], [3]. Using FFT on the collected data results information in order domain. The signals that are periodic in revolution domain appear as peaks in order domain. The key trick is that that the measurement rate tracks the rotational speed of the machine. By this way, e.g. if a vibration peak occurs three times every resolution at the same shaft angle position a peak appears at the third order in the order spectrum.

The figure below (Fig. 3) shows the method and the result of a signal sample collecting in time and by angle difference [10].

Practically, the data sampling by angle position is not a technically easy task. In principle three techniques are commonly usable:

- Shaft encoders, which are electro-optical devices and can generate even thousands of digital pulses per revolution. The pulses then can trigger sampling data directly into the data processing equipment memory.

- Some device which is tracking the rotation of the shaft and emulates the shaft angle sensor - and gives the necessary sampling trigger pulses.

- Digital resampling technique. The equipment stores the noise/vibration signal in digital form by fixed time intervals together with the signals of a precise rotation angle sensor. After collecting the primary info, the software devices are suitable to resample the noise/vibration signal from time domain to revolution domain. Probably this method is the most advantageous for simple experiments because there is no need of angle sensor. However, the numeric algorithm eventually may cause some distortion of the result.

The vibro-acoustic characteristics of engines can be studied from different viewpoints and for a number of purposes. Modern combustion engines belong to sophisticated, complicated and very expanded mechatronic systems. As sound pollution has become an important issue in today's environment, many projects are intended to analyse the noise and vibration generated by vehicle engines [7]. On the other side, one of the most sensitive parameters for monitoring the condition of engine is vibration and noise characteristics. Noise and vibration sources and eventually the related faults can be classified into two groups, namely 


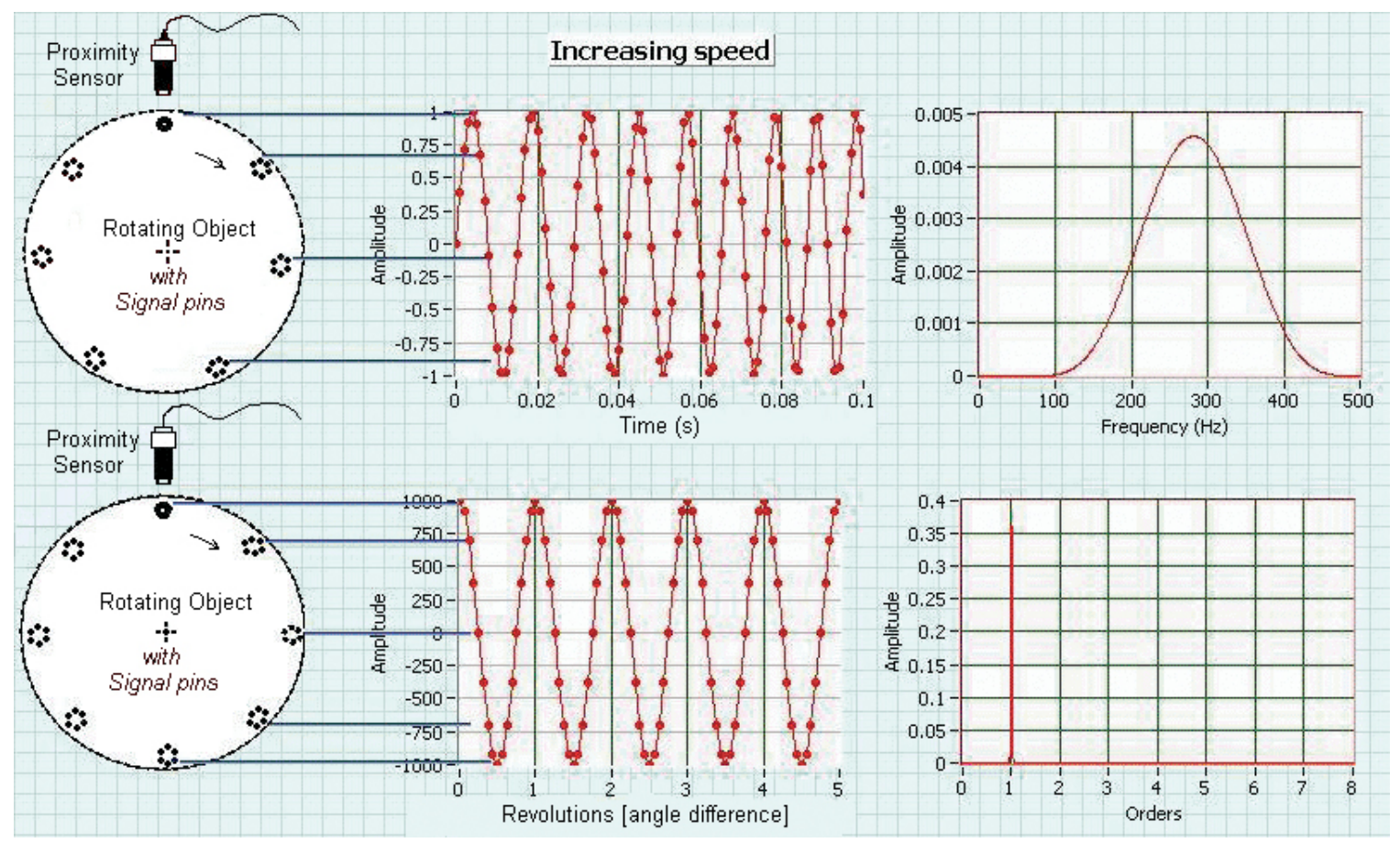

Fig. 3. Sample collecting by constant time intervals and by constant angle intervals.

the combustion process and the mechanical movements [4]. Our purpose is to develop new diagnostic methods but we can use the results of projects which basically intended to decrease the generated engine noise level.

\section{Our measurement system based on NI devices}

We developed a new measurement system which is suitable for noise/vibration diagnostics for internal combustion engines using order analysis. The system may work with or without software signal resampling. The next figure (Fig. 4) shows the system architecture.

We use an NI CompactDAQ based data acquisition and preprocessing system with the suitable $\mathrm{I} / \mathrm{O}$ modules as a cost effective solution of high technical capabilities. The system is flexible and expandable as we have eight interface module slots in the control unit. We may implement various analog, digital, timer, and counter interface tasks with HW signal preprocessing support and the LabView/LabWindows software functions. Our first tasks were the study of specific parts or sections of the engine. We used professional microphone fixed near to the possible critical noise sources and positioned the three axes vibration sensor by similar considerations. For executing order analysis we need regular angle interval signals - our solution is the usage of a ROD426 incremental rotation sensor with impulses by each $6^{\circ}$ angle revolution. It is equal to the crankshaft position sensor signal. However, to indicate the reference angle position three single pulses are missing from series of the crankshaft sensor signal. As a consequence, it seems it is not usable for the order analysis [5]. Later, in some cases we may need angle signals of higher resolution. As the rotation direction is the same every time, we can multiply the resolution of the recent sensor by two or four by HW or SW methods. One engine operation cycle consists of two crankshaft revolution and the camshaft sensor's signal can distinguish the two revolutions during one cycle. Finally, one additional simple and sure possibility is the usage of an ignition sparkle sensor to identify some engine piston working in synchrony with shaft angle position. Optionally we can synchronize our measurement to other events outside, or can give trigger signal e.g. to start video recording for more complex studies (like fast visual observations).

\section{Experimental work}

At present diagnostics project our basic aim was to detect faults of some parts or sections of the engine. A general criterion as to whether faults and abnormalities can be observed is that they appear as an indictable difference or unusual parametric value in the measured vibration or acoustic signal. There are many factors that impact the fault information content of the signals including the chosen sensors, the measurement points, shielding disturbing effects from the near and far environment etc. [6]. We basically focused on the following subjects:

- where, why and how is vibration or noise generated by the combustion engine,

- by which way, how does vibration and noise propagate trhough different materials of parts assembled together,

- how can we determine the best test positions for the sensors to capture the abnormalities [8].

We concentrated on mechanical faults. Some examples of these faults are problems with the crankshaft, faults in belt drives, e.g. damaged timing belt, camshaft defects, defective or missing connecting rod bearings, cylinder ringing, faults in oil pump, etc. Seeing that all faults which are followable by vibro-acoustic 


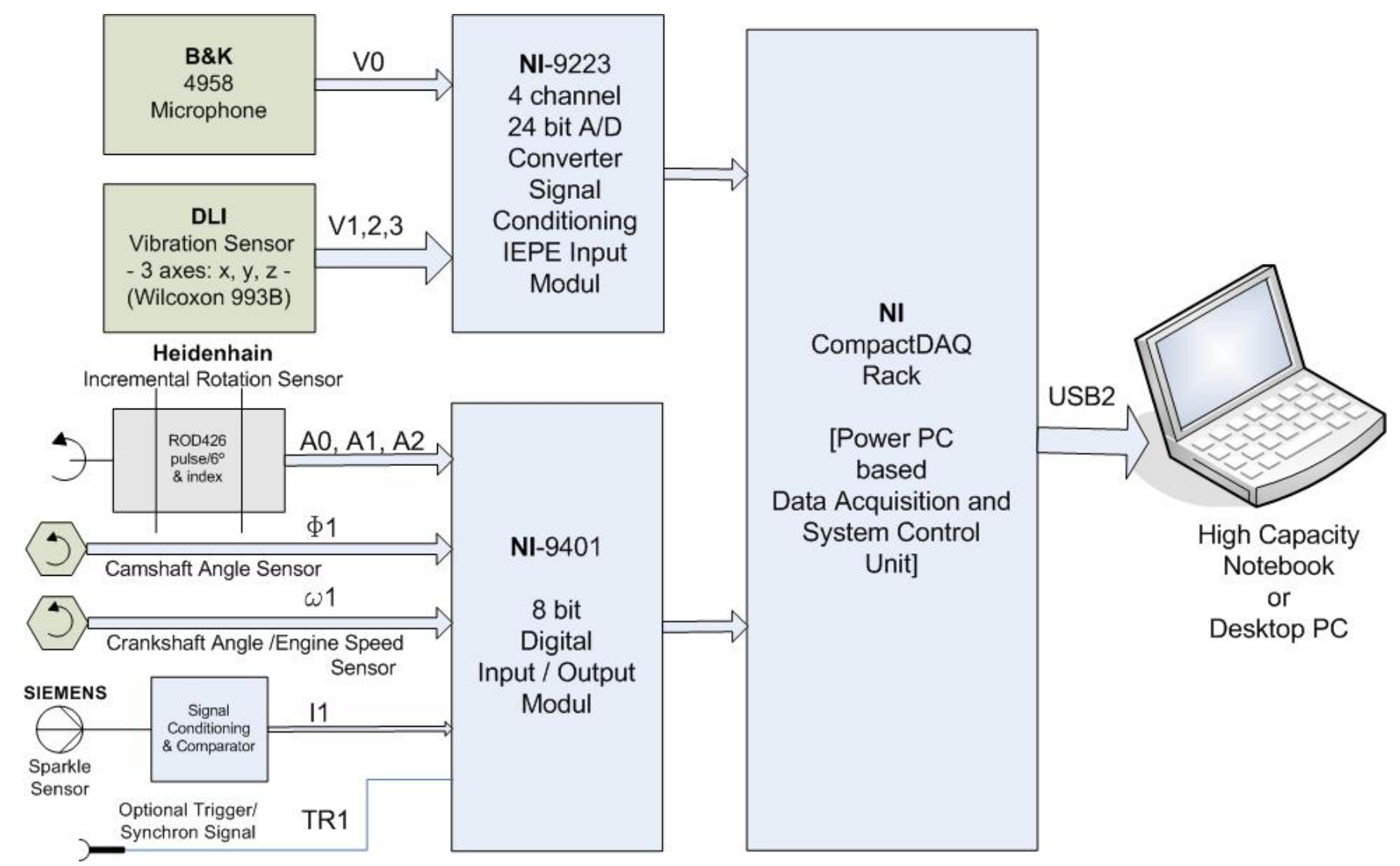

Fig. 4. The newly developed Data Acquisition System architecture

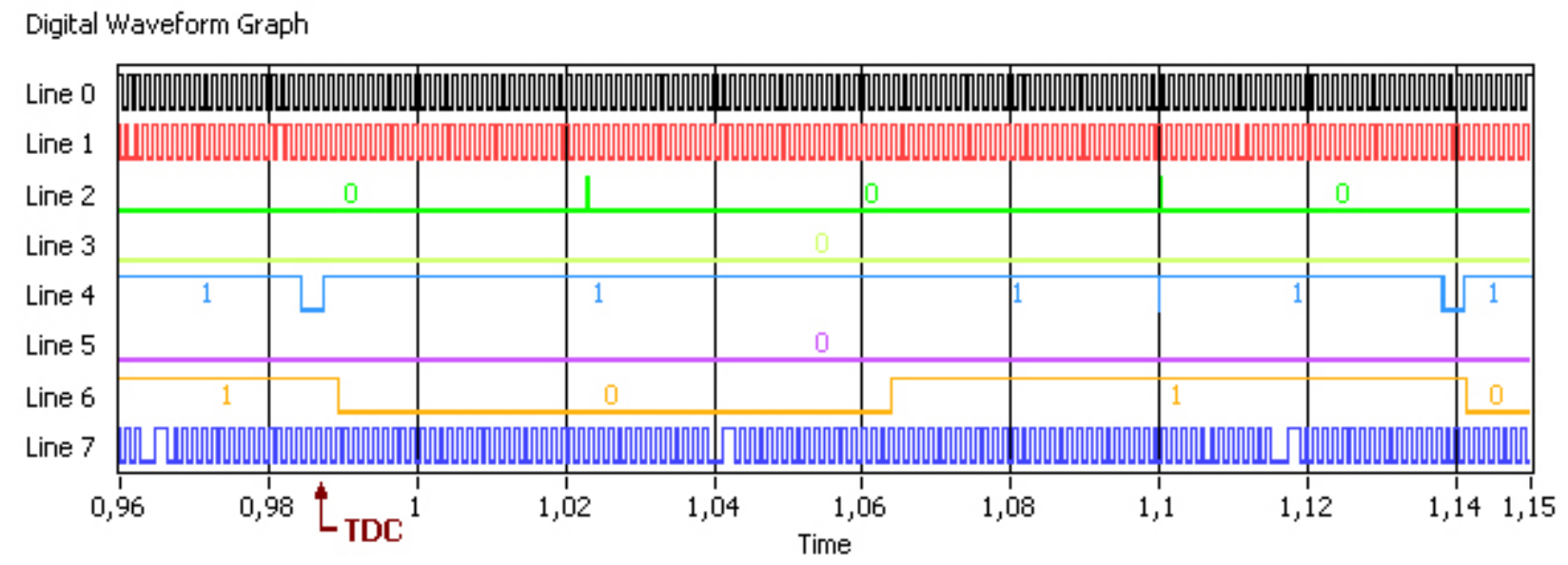

Signal marking and remarks:

Line 7: Engine Crankshaft sensor

Line 6: Engine Camshaft sensor 1

Line 5: Engine Camshaft sensor 2 (not connected here)

Line 4: Ignition Signal, Piston 1

Line 3: Trigger sensor (not used here)

Line 2: Heidenhain incremental rotation sensor Index signal (A0)

Line 1: Heidenhain incremental rotation sensor Angle signal (A1) by each $6^{\circ}$ angle movement Line 0: Heidenhain incremental rotation sensor Angle signal (A2) by each $6^{\circ}$ angle movement with $90^{\circ}$ phase shift to $\mathrm{A} 1$

TDC: Top Dead Center (in Line 7, crankshaft pulse position)

Fig. 5. Timing Relations inside the engine 


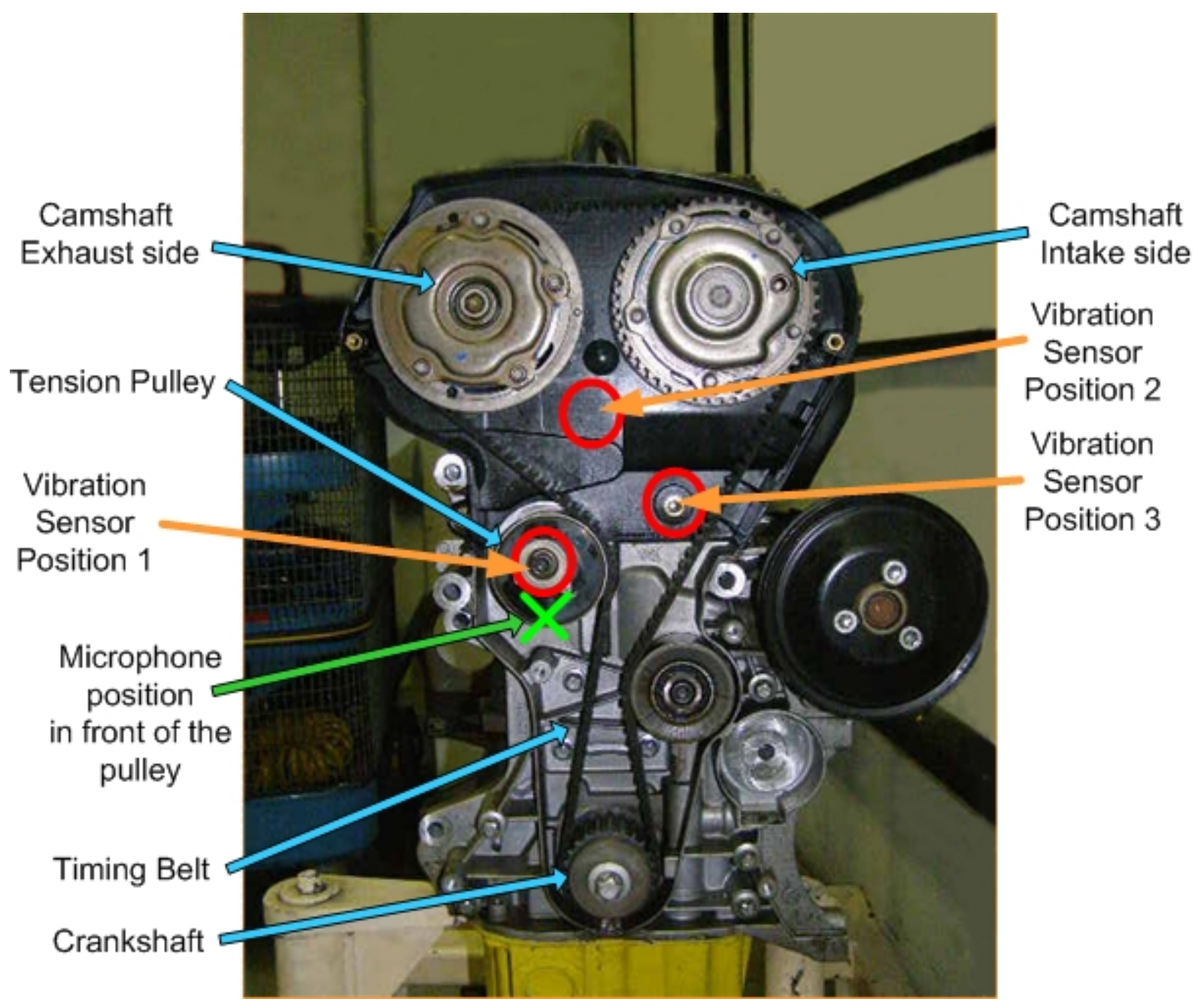

Remarks:

- The microphone was fixed on the engine test stand in front of the pulley.

- In the case of the vibration sensor we proved three positions. It seems we got the best results at Position 1, using a modified tension pulley fastening screw. At Position 2 we applied a glued support plate. At Position 3 we used a regular screw hole of the engine body.

- We connected a flexible shaft coupling among the main central screw of the crankshaft and the Heidenhain incremental angle sensor in front of the crankshaft.

Fig. 6. The front side of the engine with the tension pulley and the measuring positions
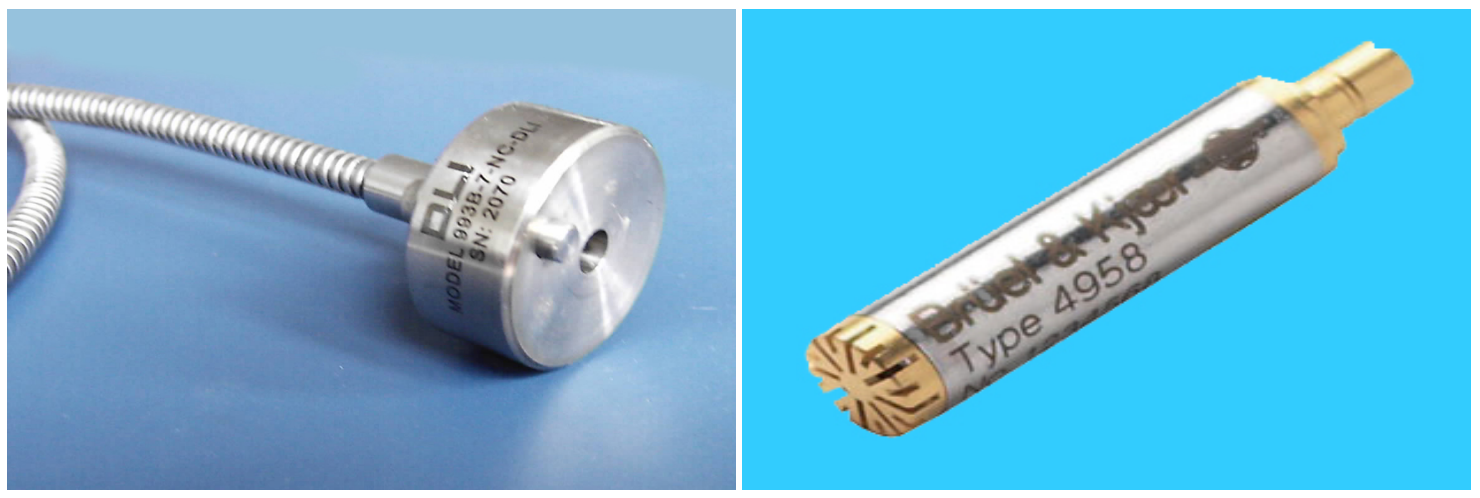

Fig. 7. The DLI 3-axes Vibration Sensor and the B\&K Precision Microphone 

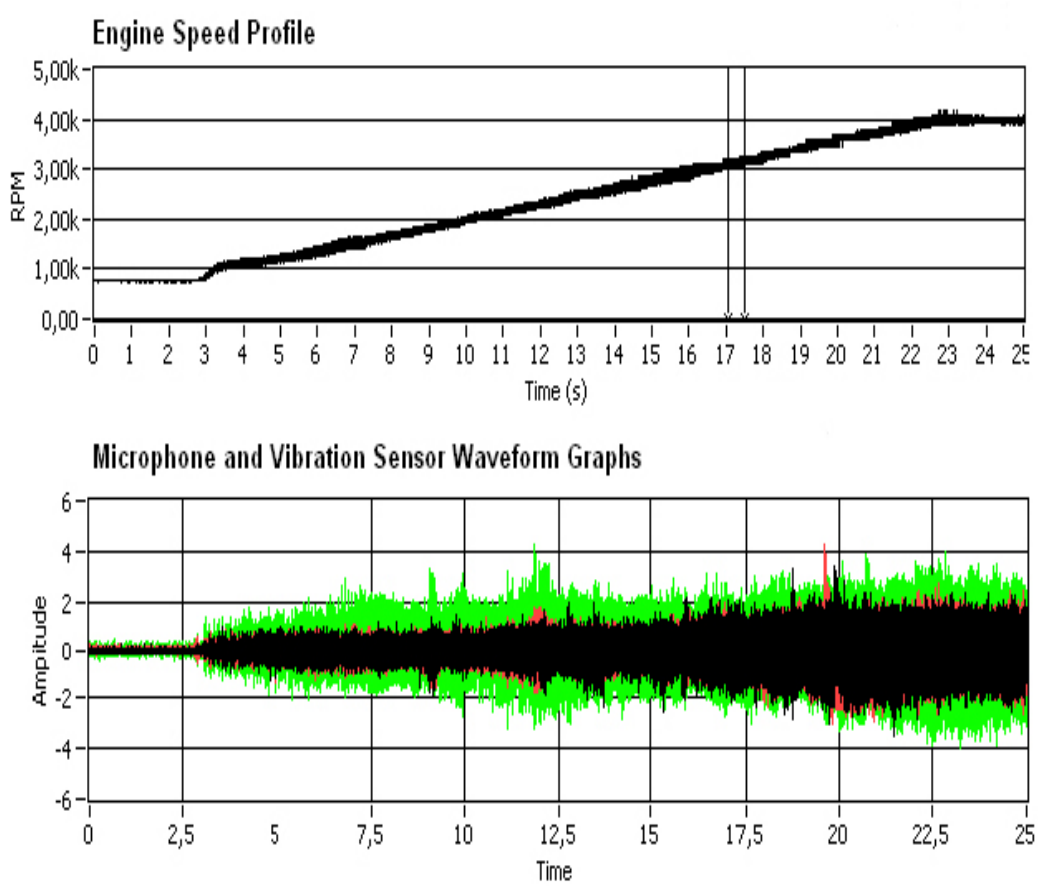

Fig. 8. Typical engine speed profile during run-up and the related vibration and noise signals

signals are related to excitation events and excitation connected to the combustion process, we made measurements to study the time relations between ignition, crankshaft and camshaft rotation. We used the digital sensors shown in Fig. 4 for this purpose. Some sample results are shown in Fig. 5 .

The measurement signals shown above make it possible to study the correlation between rotation parameters (including RPM, angle speed, shaft position, etc), ignition timing, and the vibration or noise signals. The time and other parametric relations between the signals give a great amount of information but the possibilities can be the subject of an other study.

After developing the measurement system we started to observe the behaviour of perfect and faulty parts and related symptoms. One project intended to screen tension pulleys with tension spring problems. The tension pulley makes the timing belt tight during the operation of the engine, e.g. during acceleration or deceleration. In some rare cases some pulley springs present resonance effects at some rotation speed(s) which can cause fatigue breaking. First we determined the microphone and the vibration sensor positions.

We used professional sensor devices shown in Fig.7 7

The basic characteristics of the sensors:

- Vibration Sensor, Type Wilcoxon Research 993B (manufactured especially for DLI), 3 axes

Sensitivity: $25 \mathrm{mV} / \mathrm{g}$

Frequency range: $\mathrm{Z}$ axis $-2-10.000 \mathrm{~Hz}, \mathrm{X}$ and $\mathrm{Y}$ axis -2 $-7.000 \mathrm{~Hz}$

Interface: IEPE

- Precision Array Microphone, Type B\&K4958 of Bruel\&Kjaer
Sensitivity: $12,5 \mathrm{nV} / \mathrm{Pa}$

Frequency range: $10-20.000 \mathrm{~Hz}$

Interface: IEPE, TEDS

Dynamic range $28-140 \mathrm{~dB}$

Dimensions: $34 \mathrm{~mm}$ long, $7 \mathrm{~mm}$ diameter

The selected B\&K microphone is practical in our measurements even because of the small dimensions, and it can be used in arrays which can be important in our later experiments.

\section{Diagnostic results of tension pulley test}

We made a great number of measurements testing several pulleys and some engine types. As a result we got a big data base which consists of information about the original time based and processed frequency and order domain information. The tension pulleys tests can demonstrate the system functionality and capability. In the case of pulley tests first we made screenings by sweeping the rotation speed and observed the critical values. In the other phase we observed the resonance phenomena at the critical working conditions. Some summarized results can be seen in Figs. 8 and 9 . We plan to present the results in water-fall diagram too in the next phase of the work. Note: the diagrams below can only demonstrate the results of the measurements. Running the software we can select and magnify parts of the diagrams, select or suspend signals with their colors, which makes the analysis, e.g. detailed data comparison possible or easier.

Especially in the case of the tension pulley, the resonance effect is observable in the order spectrum; however, the study of the frequency domain data is the most efficient method.

Below, in Fig. 10, the phenomena is clearly viewable at about $105 \mathrm{~Hz}$ as both the microphone and the vibration sensor axes give sharp peek values. 


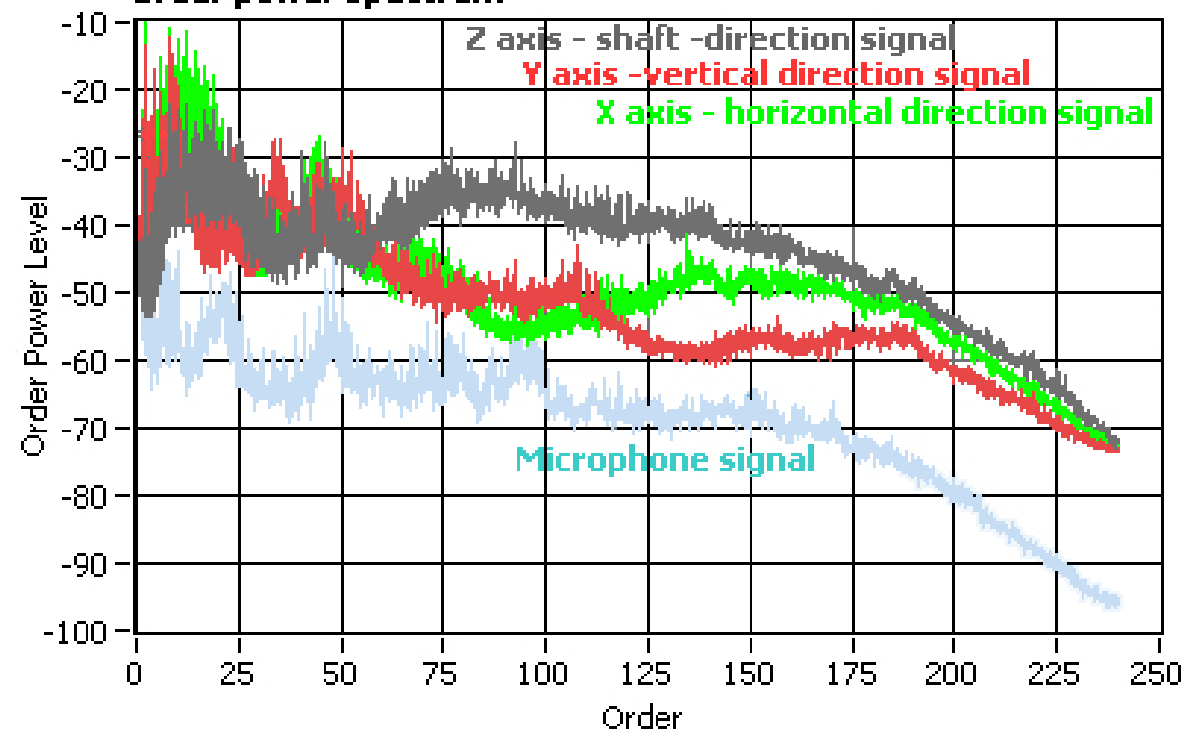

Remarks:

- The figure shows the full software capability, but for fault diagnosis the results in the left part of the diagram are important

- The "Order Power" phrase refers to the algorithm method which calculates the order spectrum from the power level of the signal in time.

- In the diagrams, the color coding (and the related grey scale tint order in black-and-white representation) is:

Light Blue: microphone

Green: vibration $X$ axis

Red: vibration $Y$ axis

Black or dark grey: vibration $Z$ axis

Fig. 9. The order power spectrum calculated from the signals above

Based on the experimental results we can design screening methods using parameter level windowing procedures to catch the faulty risky parts.

\section{Summary}

The newly developed complex "custom design" hardware and software system for vibration and acoustic noise analysis verified in practice that it is well suitable for fault diagnostics purposes. The new measurement results acquired using our flexible HW assembly and SW system also verified that modern noise and vibration diagnosis procedures are very sensitive methods. The methods can capture hidden and special problems for which even the most straightforward functional testing is not really adequate. As a result of the research, e.g. we can screen tension pulleys of engine timing belt which have fault risk during operation later because of occasional spring resonance. However, the system capability to show faulty parts is very large and makes it possible to identify risky elements with increasing diagnostic possibilities. The next steps include the enhancement of the hardware capabilities and the usage of more sophisticated software solutions and mathematical procedures. We plan to utilize the system to catch hidden fault connected problems which may be observable in frequency, or in order domain, or which are eventually detectable with combined analysis method only.
Frequency Power Spectrum

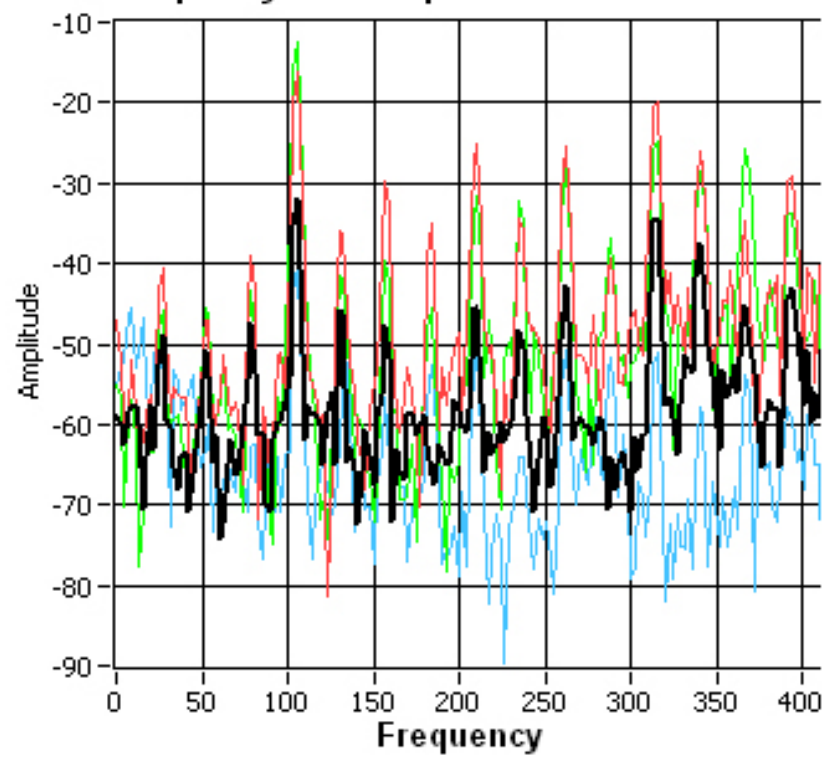

Remarks

- The system is capable to provide the frequency spectrum up to $25 \mathrm{kHz}$. but in this case data in the high frequency interval have low importance

Fig. 10. The frequency power spectrum of a potentially risky part 


\section{References}

1 Bánlaki P, Kulcsár Sz, Monitoring the Operation of Internal Combustion Engines Using Order Analysis of Noise and Vibration Data, Journal Of Machine Manufacturing XLIX (2009), 142-144.

2 Bruel \& Kjaer, What is order analysis and what is used for, B\&K, 1999, available at WWW. bksv. com

$3 \ldots$, Order analysis, B\&K, 1999, available at www.bksv.com/.../ orderanalysis.aspx

4 Denton T, Advanced Automotive Fault Diagnosis, Butterworth-Heinemann (2006), 98-121.

5 Dietse H, Automotive Sensors, Robert Bosch Gmbh, 2005.

6 Dömötör F, Rezgésdiagnosztika I, Főiskolai Kiadó, Dunaújváros, 2008.

7 Fantana NE, Study Regarding Vehicle Noise Analysis, Annales of the Oradea University, Fascicle of Managament and Technological Engineering, 2007, pp. 447-450.

8 Filep E, Mechanikai hullámok, Ábel Kiadó, Cluj-Napoca, 1999.

9 Nagy I, Technical Diagnostics I. - Vibration Analysis, Delta3N, 2007.

10 National Instruments: Understanding Order Analysis and Resampling, 2007, available at www.ni.com 